\title{
Application of RTDF to particles with curved surfaces
}

\author{
E. Hesse, D.S. Mc Call, Z. Ulanowski, C. Stopford, \\ and P.H. Kaye
University of Hertfordshire, Centre for Atmospheric and Instrumentation Research, Hatfield, Hertfordshire, UK.
tel: +44 (0) 1707 286170, fax: +44 1707 284185, e-mail: e.hesse@herts.ac.uk

\begin{abstract}
The applicability of RTDF is extended to particles with curved surfaces. This allows tests against $T$-matrix calculations for larger size parameter and modeling of light scattering by more realistic particle shapes, such as ice crystals with rounded edges.
\end{abstract}

\section{Introduction}

The importance of ice and mixed-phase clouds to the earth-atmosphere radiation balance and climate is well established. However, despite extensive study, there is still a large uncertainty over the radiative properties of these clouds. This is partly due to inadequate theoretical models of light scattering by the constituent ice crystals of realistic shapes and sizes.

Computations of light scattering properties for non-spheroidal particles based on exact methods like the Separation of Variables (SVM) Method, e.g. [1], T-matrix [2,3], Discrete Dipole Approximation (DDA) [4] have upper size parameter limits of applicability, depending on the method and the complexity of particle shape. This leaves a size parameter range that is covered neither by exact methods nor by Geometric Optics (GO). A modified Kirchhoff approximation (MKA) method has been introduced [5] to calculate far fields from classical Geometric Optics (GO) results, which encouraged the development of the Improved GO model [6]. The latter is, however, computationally expensive. For moderate values of the size parameter the Finite Difference Time Domain (FDTD) method can be used [7] but it too, puts severe demands on computational resources. Thus, despite its limitations, Geometric Optics (GO) combined with projected-area diffraction, e.g. [8], is still the most widely used model for moderate to large size parameters. Recently, diffraction on facets was introduced into a ray tracing model (Ray Tracing with Diffraction on Facets, RTDF) [9-11]. This method maintains the flexibility and computational efficiency of GO while producing much improved results. Given the rapid and flexible computation offered by raytracing based models, it is possible to create 2D light scattering patterns for even very complex crystals. Such patterns provide much more information than azimuthally averaged scattering data such as a phase function. In contrast to standard GO, the RTDF model can produce such patterns for fixed as well as averaged random orientation. 2D scattering patterns have been correctly predicted by the RTDF model [10], and it is therefore expected to become a suitable tool for particle characterization, in particular since cloud probes now exist that characterize ice particles on the basis of 2D scattering patterns (e.g. [12]). A large proportion of ice crystals have curved as well as planar surface components, e.g. due to the onset of sublimation or melting. Other atmospheric particles, like Saharan dust, are irregular. Their surfaces could be approximated as multifaceted objects. In this paper, the RTDF model is used to model scattering by 'hybrid' particles, the surface of which has both planar and curved components. RTDF phase functions of circular cylinders are compared with $T$-matrix [13], which is an analytical technique, and GO combined with projected area diffraction [8]. The effect of rounded edges on 2D scattering patterns of hexagonal columns is demonstrated. 


\section{Method and results}

The RTDF model combines ray tracing with diffraction on flat facets. The model calculates diffraction using an approximation for the far-field direction of the Poynting vector. The angle of diffraction of a reflected or refracted ray is calculated from the ray's proximity to the facet edges [11]. In the following, curved surfaces will be approximated by arrays of planar facets. As an example, the lateral surface of a circular cylinder will be approximated by a succession of thin stripes. The approximation of a curved surface will improve with the number of plane facet elements, avoiding artificial sharp edges and therefore reducing artefact structures in the angular distribution of scattered light. A decrease of the angle between surface normals of neighbouring facets needs to be accompanied by a reduction of diffraction at these facets. In the following, the far field deflection angle $\varphi_{o}$ calculated by RTDF [11] is multiplied by the sine of the angle $y$ between the normals of the neighbouring facets under consideration, if $y<90^{\circ}$.

$$
\begin{array}{ll}
\varphi=\varphi_{o} \sin \gamma & \forall 0<\gamma<\pi / 2 \\
\varphi=\varphi_{o} & \forall \pi / 2<\gamma<\pi
\end{array}
$$

This ensures that no diffraction takes place between coplanar facets. Full far field diffraction is applied between facets with normals which include an angle of $90^{\circ}$ or larger.

The RTDF results are compared with computations using $T$-matrix $[13,14]$, which is an analytical technique, and GO combined with projected area diffraction [8]. Fig. 1 shows randomized phase functions for a circular cylinder with refractive index 1.311 for size parameters $x=2 \pi a_{\text {sph }} / \lambda$ equal to 40,80 and 120 , where $a_{s p h}$ is the radius of a sphere with equivalent surface area. The aspect ratio of the column $L / 2 a$ is 1 , where $L$ is the column length and $2 a$ its diameter.
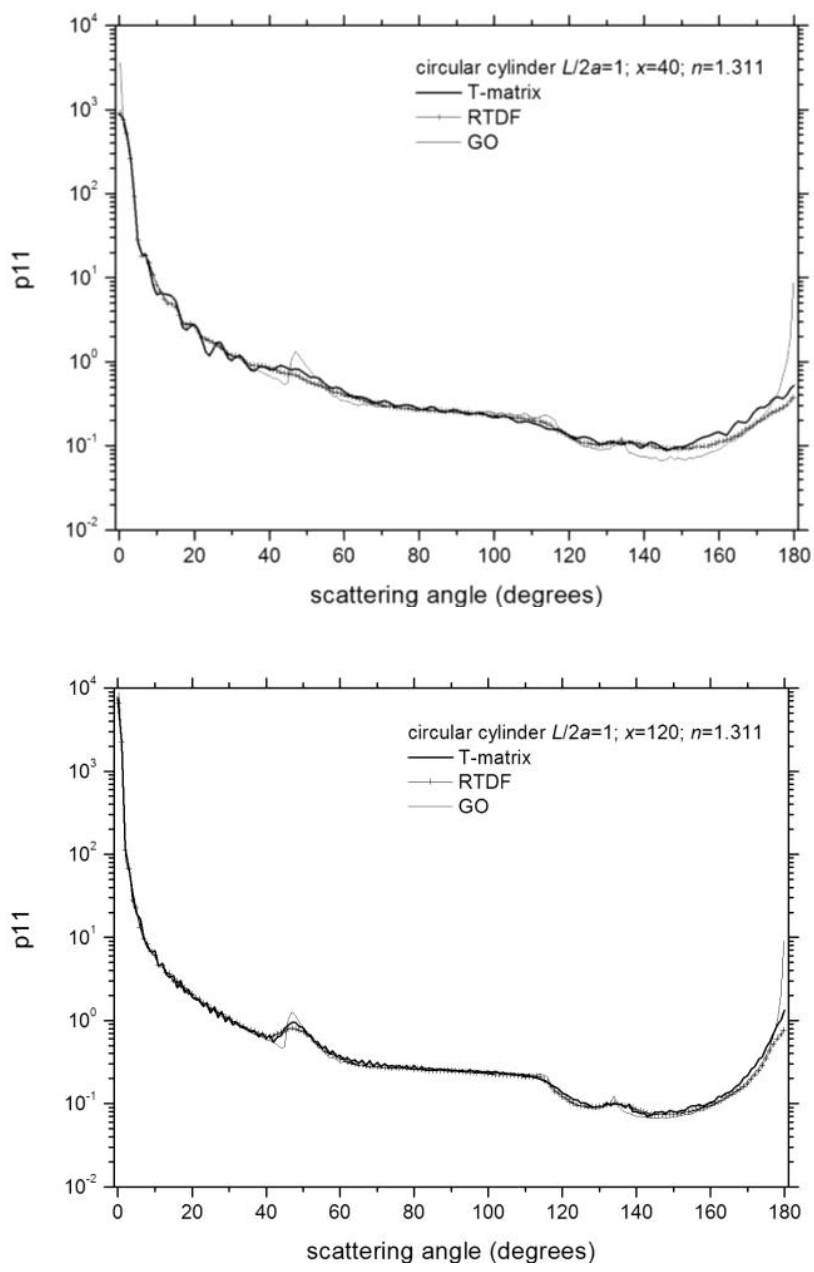

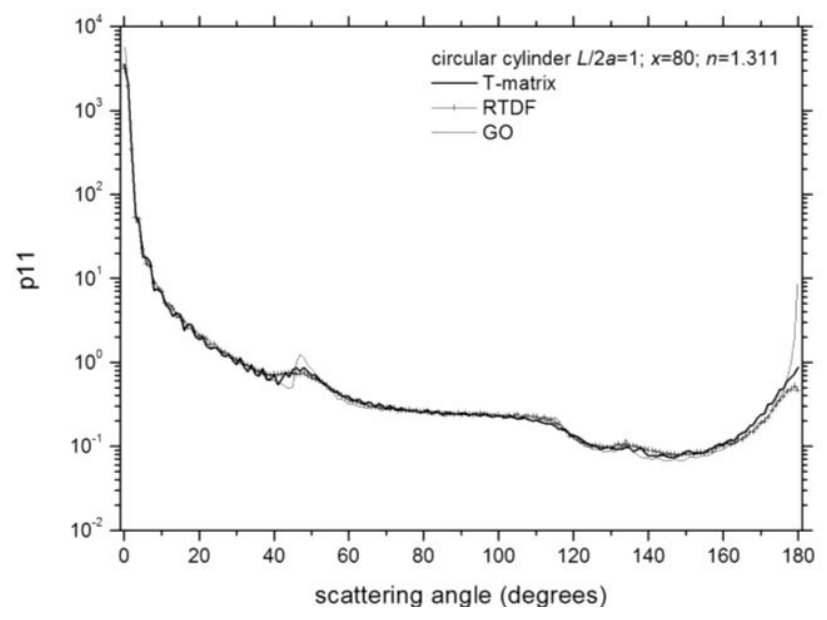

Figure 1: Randomized phase functions for circular cylinders with refractive index $n=1.311$, aspect ratio $L / 2 a=1$ and size parameters $x=2 \pi a_{\text {sph }} / \lambda$ of (a) $x=40$, (b) $x=80$ and (c) $x=120$, respectively, calculated using RTDF in comparison with $T$-matrix $[13,14]$ and GO with projected area diffraction [8] results. 
The RTDF results approximate the $T$-matrix calculations much better than $\mathrm{GO}$ over the whole angular range, and in particular in near exact forward and backscattering, in the halo region, and in the backscattering region. In agreement with the T-matrix calculations [13], the RTDF results show that size parameter 40 is too small to produce a pronounced $46^{\circ}$ halo. Diffraction features appear slightly wider in RTDF than in the T-matrix results due to the approximation of diffraction during internal reflection and refraction steps by far field values (multiplied by $\sin y$ according to eq.(1)), currently implemented in the RTDF model [11].

Fig. $2 a$ shows $2 \mathrm{D}$ scattering patterns calculated for a hexagonal column of size parameter $2 \pi a / \lambda$, where $a$ is the edge length of the basal facet, aspect ratio $L / 2 a=3$ and orientation described by Euler angles $\alpha=0^{\circ}, \beta=70^{\circ}, \gamma=10^{\circ}$, as seen from the perspective of the incident beam. Fig. $2 b$ shows the pattern for the same column but with rounded long edges of the prism facets. The circumscribing circle has a radius of 0.956 a. Rounded edges are approximated by a succession of eight thin facets, each of which covers an angular segment of $1^{\circ}$ around the long axis of the particle.

The main feature in both scattering patterns is the bright arc through forward scattering. In Fig. 2a three bright spots are situated on the arc. The central one is essentially due to rays entering through facet 4 and leaving through facet 7 (see Fig. 2a for facet numbers). The two outer ones are due to refraction through $60^{\circ}$ prisms, entering through facets 5 and 3 respectively, and leaving through facet 7 . The additional bright spots along the arc in Fig. $2 \mathrm{~b}$ are due to rays passing through edge facets. The double features left and right of the central bright spot are due to rays entering through facet 4 and leaving through the (rounded) edge between facets 6 and 7, and rays entering at the edge between facets 3 and 4 and leaving through facet 7 , respectively. The splitting of these spots is due to the approximation of the curved edge by a discrete number of plane facets. In Figs. $2 a$ and $b$, the central spot on the arc is connected to one bright spot almost directly above, corresponding to refraction through facets 1 and 7 . In Fig $2 \mathrm{~b}$, an additional bright spot slightly to the left of the previous one is due to rays refracted through facet 1 and the edge between facets 6 and 7. Below each of the three maxima on the arc in Fig. 2a is another intensity maximum due to more complicated ray paths entering through facet 5 , 4 , and 3 , respectively. (The central lower maximum is stronger than the outer ones due to contributions by external reflection from facet 1.) In Fig. $2 \mathrm{~b}$ we find corresponding maxima to each intensity maximum along the arc caused by ray paths through edge facets.
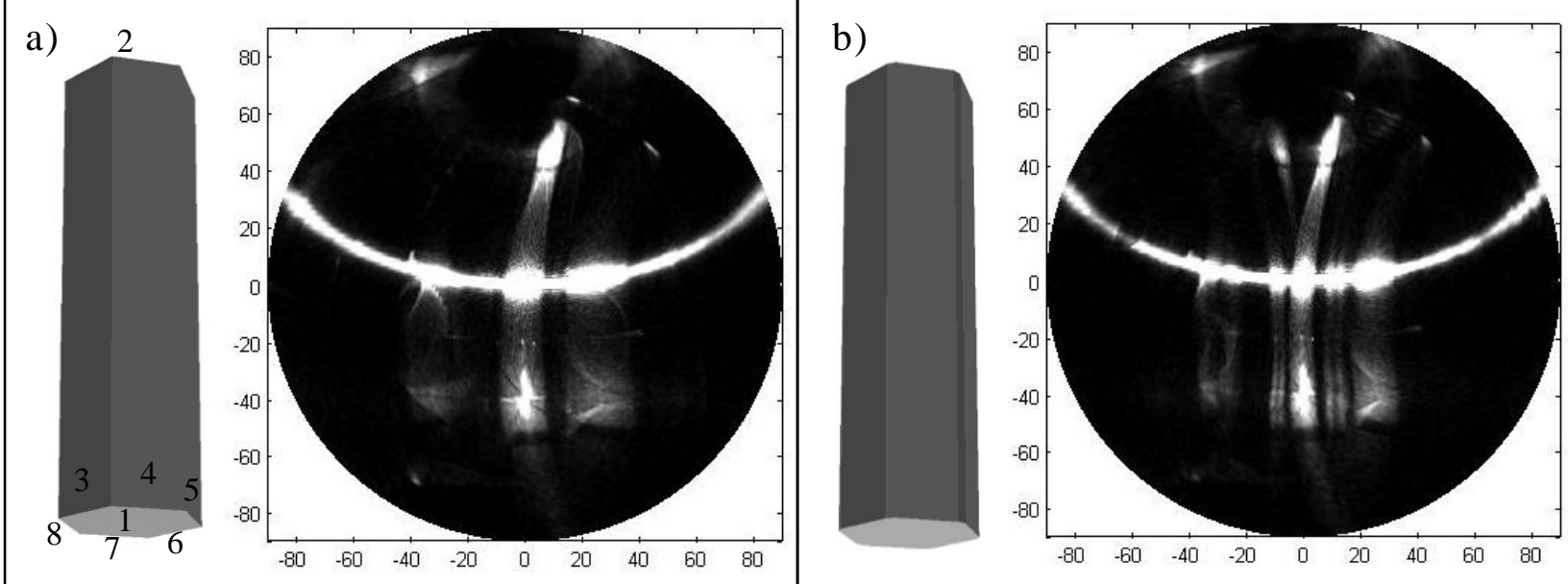

Figure 2: a) Orientation of a hexagonal column $\left(\alpha=0^{\circ}, B=70^{\circ}, y=10^{\circ}\right)$ from the point of view of the incident light and scattering pattern projected into the forward facing hemisphere; the facets are numbered as shown. b) Schematic and scattering pattern for the same column as in Fig. a), but with rounded long edges of the prism facets.

\section{Conclusion}

The RTDF model has been developed further to approximate curved surfaces by an array of planar facets and taking into account the angle between surface normals when calculating diffraction during ray-tracing. 
The enhanced RTDF model has been tested against $T$-matrix results for circular cylinders. The RTDF results approximate the $T$-matrix calculations much better than $\mathrm{GO}$ over the whole angular range, and in particular in near-exact forward and backscattering, and in the halo region. The modification of 2D scattering patterns by rounded edges in comparison to pristine crystals has been demonstrated for a hexagonal column. The expansion of the applicability of the RTDF model towards particles with curved surfaces and 'hybrid particles', which have plane as well as curved surfaces is potentially useful for the calculation of the scattering properties of ice crystals of realistic shapes and comparison with experimental data.

\section{Acknowledgment}

This research was supported by the Natural Environment Research Council of the UK.

\section{References}

[1] T. Rother, K. Schmidt, S. Havemann, "Light scattering on hexagonal ice columns", J Opt Soc Am A 18, 2512-2517 (2001).

[2] M.I. Mishchenko, G. Videen, V.A. Babenko, N.G. Khlebtsov, T. Wriedt, "T-matrix theory of electromagnetic scattering by particles and its applications: a comprehensive reference database", J Quantit Spectrosc Radiat Transf 88, 357-406 (2004).

[3] M.I. Mishchenko, G. Videen, V.A. Babenko, N.G. Khlebtsov, T. Wriedt, "Comprehensive T-matrix reference database: A 2004-06 update", J Quantit Spectrosc Radiat Transf 106, 304-324 (2007).

[4] M.A. Yurkin, A.G. Hoekstra, "The discrete dipole approximation: An overview and recent developments", J Quantit Spectrosc Radiat Transf 106, 558-589 (2007).

[5] Muinonen K. "Scattering of light by crystals: a modified Kirchhoff approximation", Appl Opt 28, 3044-3050 (1989).

[6] P. Yang, K.N. Liou "Geometric-optics-integral equation method for light scattering by nonspherical ice crystals", Appl Opt 35, 6568-6584 (1996).

[7] P. Yang, K.N. Liou. In: Mishchenko MI, Hovenier JW, Travis LD, editors. Light scattering by nonspherical particles, (New York: Academic Press, 1999. p. 173-221).

[8] A. Macke, J. Mueller, E. Raschke, "Single scattering properties of atmospheric ice crystals", J Atmos Sci 53, 2813-2825 (1996).

[9] E. Hesse, Z. Ulanowski, Scattering from long prisms using ray tracing combined with diffraction on facets. J Quantit Spectrosc Radiat Transf. 79-80C, 721-732 (2003)

[10] A.J.M. Clarke, E. Hesse, Z. Ulanowski and P.H. Kaye, "A 3D implementation of ray-tracing with diffraction on facets: Verification and a potential application", J Quantit Spectrosc Radiat Transf 100, 103-114 (2006).

[11] E. Hesse, "Modelling diffraction during ray-tracing using the concept of energy flow lines", J. Quantit. Spectrosc. Radiat. Transf. 109, 1374-1383 (2008).

[12] P.H. Kaye, E. Hirst, R.S. Greenaway, Z. Ulanowski, E. Hesse, P.J. DeMott, C. Saunders, P. Connely, "Classifying atmospheric light scattering by spatial light scattering", submitted to Optics Letters.

[13] M. I. Mishchenko, A. Macke, "How big should hexagonal ice crystals be to produce halos?", Appl. Opt. 38, 1626-1629 (1999).

[14] M.I. Mishchenko and L.D. Travis, "Capabilities and limitations of a current FORTRAN implementation of the T-matrix method for randomly oriented, rotationally symmetric scatterers", J. Quant. Spectrosc. Radiat. Transfer 60, 309-324 (1998). 